\title{
Alpha-induced astrophysical reactions studied at CRIB
}

\section{H. Yamaguchi*}

Center for Nuclear Study, Graduate School of Science, University of Tokyo 2-1 Hirosawa, Wako, Saitama 351-0198, Japan

E-mail: yamag@ens.s.u-tokyo.ac.jp

T. Hashimoto, S. Hayakawa, D.N. Binh, D. Kahl, S. Kubono

Center for Nuclear Study, Graduate School of Science, University of Tokyo

2-1 Hirosawa, Wako, Saitama 351-0198, Japan

CRIB (CNS Radioactive Ion Beam separator) is a low-energy RI beam separator at the Center for Nuclear Study (CNS) of the University of Tokyo. Studies on proton and alpha resonance scatterings, $(\alpha, p)$ reactions, and other types of measurements ( $\beta$-decay lifetimes etc.) have been performed using RI beams at CRIB, motivated by interests on astrophysical reactions and exotic nuclear structure. Among the studies at CRIB, the measurement of ${ }^{7} \mathrm{Li}+\alpha$ resonant scattering is presented.

11th Symposium on Nuclei in the Cosmos, NIC XI

July 19-23, 2010

Heidelberg, Germany

${ }^{*}$ Speaker. 


\section{Introduction}

CRIB $[1,2]$ is a radio-isotope (RI) beam separator of CNS, University of Tokyo, located in the RIBF facility of RIKEN Nishina Center. CRIB can produce low-energy $(<10 \mathrm{MeV} / \mathrm{u}) \mathrm{RI}$ beams in flight, using primary heavy-ion beams from the AVF cyclotron of RIKEN $(K=70)$. RI beams are produced via direct reactions such as $(p, n),(d, p)$, and $\left({ }^{3} \mathrm{He}, n\right)$, taking place at an 8-cm-long gas target with a maximum pressure of 760 Torr. Solid targets such as beryllium can also be used as the production target. The intensity of the secondary beam is typically $10^{4}-10^{6} \mathrm{pps}$. For a more intense RI beam production, a cryogenic target system, in which the target gas can be cooled down to about $90 \mathrm{~K}$, is currently available at CRIB. For example, a ${ }^{7} \mathrm{Be}$ beam of $2 \times 10^{8}$ pps was produced using the cryogenic target system [3]. The secondary beam is purified with an magnetic analysis using dipole magnets, and with a Wien filter, which can separate the beams according to their velocities. For relatively light RI beams such as ${ }^{7} \mathrm{Be}$, we can obtain a purity of almost $100 \%$ after the Wien filter.

A major topic of our interest is alpha-induced reactions, which can be studied using heavy-ion beams and a helium gas target. Several $(\alpha, p)$ reactions, such as ${ }^{14} \mathrm{O}(\alpha, p)[4],{ }^{11} \mathrm{C}(\alpha, p),{ }^{21} \mathrm{Na}(\alpha$, $p),{ }^{18} \mathrm{Ne}(\alpha, p)$, and ${ }^{30} \mathrm{~S}(\alpha, p)$ have been measured at CRIB. The elastic resonant scatterings of alpha particle using ${ }^{14} \mathrm{O},{ }^{21} \mathrm{Na},{ }^{7} \mathrm{Li}$ and ${ }^{7} \mathrm{Be}$ beams have also been measured. These measurements are related to astrophysical $(\alpha, \gamma)$ reaction rates, and are relevant for studying nuclear cluster structure. Among the studies, the one on ${ }^{7} \mathrm{Li}+\alpha$ measurement, which is related to the cluster structure of ${ }^{11} \mathrm{~B}$, is presented below.

The exotic cluster structures in the ${ }^{11} \mathrm{~B}$ and ${ }^{11} \mathrm{C}$ nuclei are attracting much attention in recent years [5]. The $3 / 2_{3}^{-}$state in ${ }^{11} \mathrm{~B}$ at the excitation energy $E_{\mathrm{ex}}=8.56 \mathrm{MeV}$ is regarded as a dilute cluster state [6], where two $\alpha$ particles and ${ }^{3} \mathrm{He}$ are weakly interacting. In particular, the alpha cluster structure in ${ }^{11} \mathrm{~B}$ was studied by measuring its isoscalar monopole and quadrupole strengths in the ${ }^{11} \mathrm{~B}\left(d, d^{\prime}\right)$ reaction, and the $8.56-\mathrm{MeV}$ state was suggested to have a dilute cluster structure [7,8]. A natural method to study the $\alpha$ cluster structure of ${ }^{11} \mathrm{~B}$ is to form such a cluster state by the elastic scattering of ${ }^{7} \mathrm{Li}$ and $\alpha$.

The ${ }^{7} \mathrm{Li}+\alpha$ system is also related to the astrophysical reaction ${ }^{7} \mathrm{Li}(\alpha, \gamma){ }^{11} \mathrm{~B}$. In the low temperature stellar environment which ignites the $p$ - $p$ chains, ${ }^{7} \mathrm{Li}$ is destroyed via the ${ }^{7} \mathrm{Li}(p, \alpha){ }^{4} \mathrm{He}$ reaction, and the ${ }^{7} \operatorname{Li}(\alpha, \gamma){ }^{11} \mathrm{~B}$ reaction is much weaker in comparison. However, in some high temperature phenomena, the ${ }^{7} \operatorname{Li}(\alpha, \gamma){ }^{11} \mathrm{~B}$ reaction should play important roles. For example, in the $v$-process in core-collapse supernovae [9] ${ }^{11} \mathrm{~B}$ is mainly produced via ${ }^{4} \mathrm{He}\left(v, v^{\prime} p\right)^{3} \mathrm{H}(\alpha, \gamma){ }^{7} \mathrm{Li}(\alpha$, $\gamma)^{11} \mathrm{~B}$, but the production can be enhanced with the ${ }^{12} \mathrm{C}\left(\overline{v_{e}}, e^{+} n\right)^{11} \mathrm{~B}$ reaction. A precise comparison between the observed abundance ratio of ${ }^{11} \mathrm{~B} /{ }^{7} \mathrm{Li}$ in stars and a calculation using the experimental ${ }^{7} \mathrm{Li}(\alpha, \gamma){ }^{11} \mathrm{~B}$ reaction rate may provide a constraint on the neutrino mixing parameter $\theta_{13}$, as suggested in Ref. [9].

\section{Experimental Method}

In many measurements we used the thick-target method in inverse kinematics [10], which is suitable for the low-energy beams at CRIB. In that method, the beam energy is degraded in a thick reaction target, and reactions occur at various center-of-mass energies. We detect light par- 


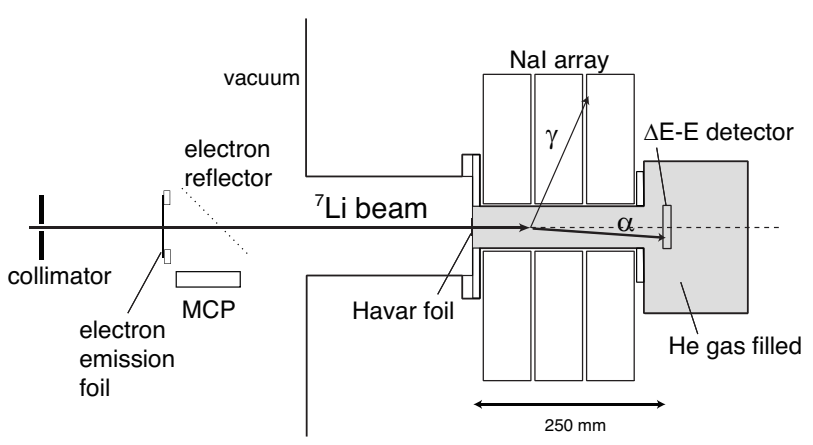

Figure 1: Arrangement of the detectors and targets in ${ }^{7} \mathrm{Li}+\alpha$ measurement.

ticles emitted after reactions, and reconstruct the kinematics. This method has several advantages, namely, (a) we can perform simultaneous measurements of cross section of various excitation energies without varying the incoming beam energy, and (b) we can perform measurement at $180^{\circ}$ in center-of-mass angle, where the Coulomb scattering is minimal. Many fruitful results have been obtained at CRIB with this method [4,11-15].

Figure 1 schematically shows the experimental setup for the ${ }^{7} \mathrm{Li}+\alpha$ scattering measurement. A ${ }^{7} \mathrm{Li}$ beam was accelerated at the AVF cyclotron and transported to the final focal plane (F3) of CRIB. The beam had a well-defined energy of $13.7 \mathrm{MeV}$, and collimated by a $3 \times 3$-mm square aperture at F3. An MCP (Micro-Channel Plate) was used to measure the position and timing of the beam. A CsI-evaporated 0.7- $\mu \mathrm{m}$-thick aluminum foil was placed on the beam axis for the secondary electron emission. The secondary electrons were reflected by $90^{\circ}$ at a biased thin-wire reflector and detected at the MCP with a delay-line readout. The gas target consisted of a 50-mmdiameter duct and a small chamber. The helium gas was housed in a $50 \mathrm{~mm}$ diameter duct and a small target chamber. The helium gas was at 920 Torr and sealed with a thin $(2.5 \mu \mathrm{m})$ Havar foil as a beam entrance window. Alpha particles recoiling to the forward angles in the laboratory frame were detected by the " $\Delta E-E$ detector", which consisted of $20-\mu \mathrm{m}$ - and $480-\mu \mathrm{m}$-thick silicon-strip detectors, placed directly in the gas chamber. The effective area of the detector was $20 \mathrm{~cm}^{2}$. The silicon-strip detectors had a position sensitivity of $3 \mathrm{~mm}$. The effective target length, namely, the distance from the beam entrance window to the detector, was $250 \mathrm{~mm}$. To measure $478-\mathrm{keV}$ gamma rays from inelastic scattering to the first excited state of ${ }^{7} \mathrm{Li}, \mathrm{NaI}$ detectors were placed around the duct. We used ten NaI crystals, each with a geometry of $50 \times 50 \times 100 \mathrm{~mm}^{3}$. They covered $20-60 \%$ of the total solid angle, depending on the reaction position in the target. The photopeak efficiency of the NaI detectors were measured at various positions in the gas target, using gammaray sources. The measurement was performed for $2.9 \times 10^{10}{ }^{7} \mathrm{Li}$ particles injected into the gas target over 60 hours.

\section{Experimental results}

Our measurement corresponds to $E_{\mathrm{ex}}=10-13 \mathrm{MeV}$ and the center-of-mass angles of $160-180^{\circ}$. The excited states of ${ }^{11} \mathrm{~B}$ in this energy region have been studied by ${ }^{7} \mathrm{Li}+\alpha$ elastic scattering $[16$, 
17] or using other methods [18]; however, some of the resonance parameters are still uncertain. Especially, the alpha widths for most of the resonances were not determined with a good precision. Using inverse kinematics, the excitation function around $180^{\circ}$ in the center-of-mass system was measured for the first time.
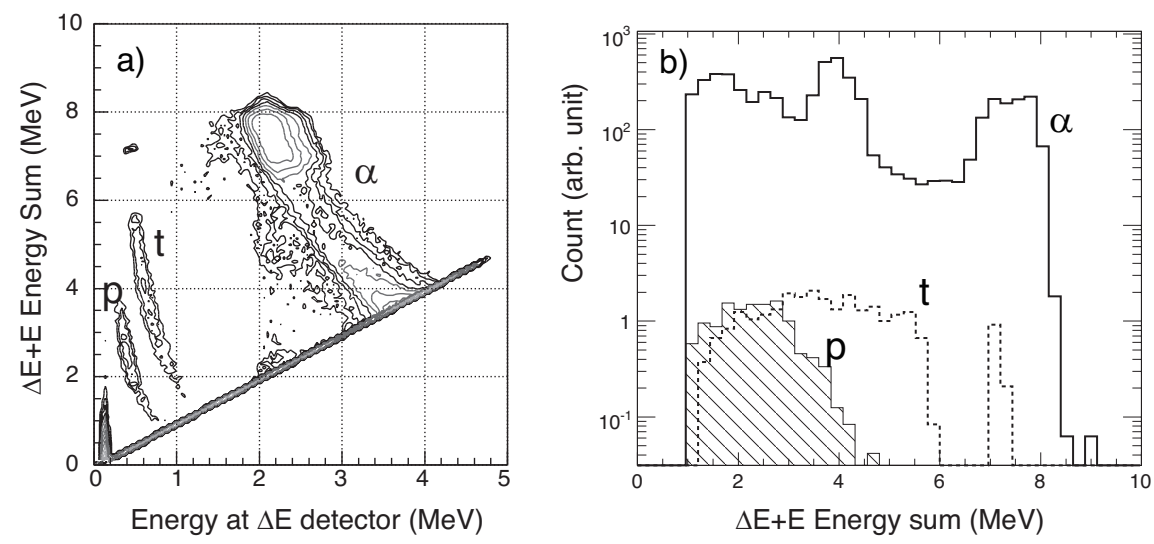

Figure 2: a) Two dimensional energy plot for the $\Delta E-E$ counter for particle identification. b) Energy spectra of the alpha, proton and triton for $>1 \mathrm{MeV}$.

The particle identification performed with the $\Delta E-E$ counter is shown as a two-dimensional energy plot in Figure 2a. As illustrated, $\alpha$ particles, protons, and tritons were clearly separated. Since the gas target was sufficiently thick to stop the ${ }^{7} \mathrm{Li}$ beam, particles heavier than lithium were not expected to reach the detector, even if produced. $\alpha$ particles from the elastic scattering were dominant in the measured particles, while comparably small numbers of protons and tritons, possibly from the ${ }^{7} \mathrm{Li}(\alpha, p)$ reaction and break up of ${ }^{7} \mathrm{Li}$ respectively, were also observed, as compared in Figure 2b. Considering that there is no strong reaction to produce ${ }^{3} \mathrm{He}$, the detected particles which deposited energy more than $1 \mathrm{MeV}$ on the first layer should be mostly $\alpha$ particles.

By kinematical calculations with a consideration of the energy loss in the gas target, the measured energy of the $\alpha$ particle was converted to a center-of-mass energy for the ${ }^{7} \mathrm{Li}+\alpha$ system, and an excitation function was obtained. The excitation function in the present work is shown in Fig. 3 with previous results [16,17], and a structure having peaks consistent with previous elastic scattering measurements $[16,17]$ was observed. New information on the resonance parameters of ${ }^{11} \mathrm{~B}$, especially on the alpha resonant widths, are expected to be obtained with our data.

\section{Summary}

We performed a measurement of ${ }^{7} \mathrm{Li}+\alpha$ elastic resonant scattering using the thick-target method in inverse kinematics for the first time. The resonance parameters to be determined in this study, such as the spin, parity and alpha width,would provide valuable information for the $\alpha$-cluster structure in the high excited states, and the astrophysical ${ }^{7} \operatorname{Li}(\alpha, \gamma)$ reaction rate in high-temperature phenomena. 


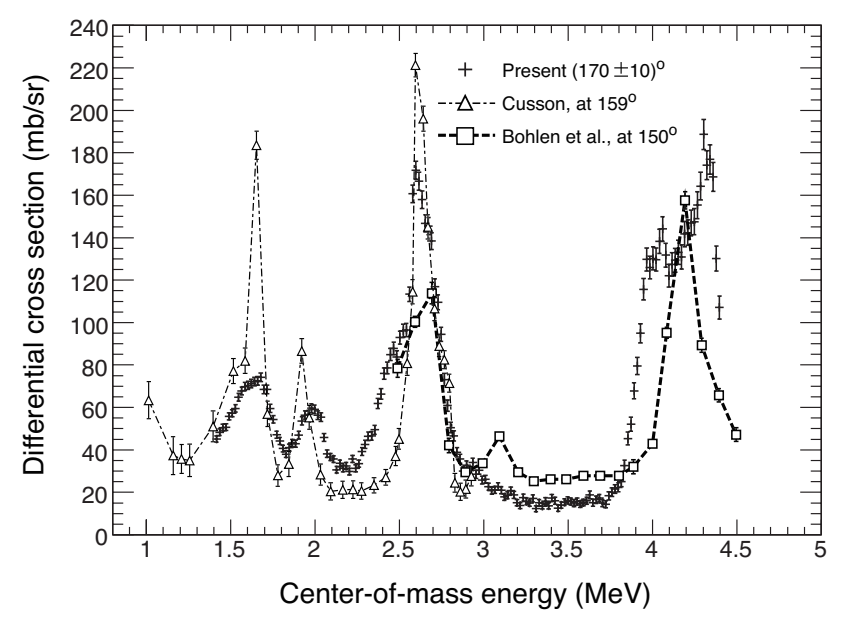

Figure 3: Excitation function of the ${ }^{7} \mathrm{Li}+\alpha$ elastic scattering cross section, compared with previous measurements $[16,17]$.

The experiment was performed at RI Beam Factory operated by RIKEN Nishina Center and CNS, the University of Tokyo. We are grateful to the RIKEN and CNS accelerator staff for their help. This work was partly supported by JSPS KAKENHI (No. 21340053) from the Ministry of Education, Culture, Sports, Science and Technology (MEXT) of Japan.

\section{References}

[1] S. Kubono et al., Eur. Phys. J. A13 (2002) 217.

[2] Y. Yanagisawa et al., Nucl. Instrum. Meth. Phys. Res., Sect. A 539 (2005) 74-83.

[3] H. Yamaguchi et al., Nucl. Instrum. Meth. Phys. Res., Sect. A 589 (2008) 150-156.

[4] M. Notani et al., Nucl. Phys. A 746 (2004) 113c-117c.

[5] Y. Kanada-En'yo, Phys. Rev. C 75 (2007) 024302.

[6] A. Tohsaki, H. Horiuchi, P. Schuck, and G. Röpke, Phys. Rev. Lett. 87 (2001) 192501.

[7] T. Kawabata et al., Phys. Rev. C 70 (2004) 034318.

[8] T. Kawabata et al., Phys. Lett. B 646 (2007) 6-11.

[9] T. Yoshida et al., Phys. Rev. Lett. 96 (2006) 091101.

[10] K. P. Artemov et al., Sov. J. Nucl. Phys 52 (1990) 408.

[11] T. Teranishi et al., Phys. Lett. B 556 (2003) 27-32.

[12] T. Teranishi et al., Phys. Lett. B 650 (2007) 129-134.

[13] J. J. He et al., Phys. Rev. C 76 (2007) 055802.

[14] H. Yamaguchi et al., Phys. Lett. B 672 (2009) 230-234.

[15] J. J. He et al., Phys. Rev. C 80 (2009) 015801.

[16] R. Cusson, Nucl. Phys. 86 (1966) 481-508. 
[17] H. Bohlen, N. Marquardt, W. von Oertzen, and P. Gorodetzky, Nucl. Phys. A 179 (1972) 504-512.

[18] F. Ajzenberg-Selove, Nucl. Phys. A 506 (1990) 1. 